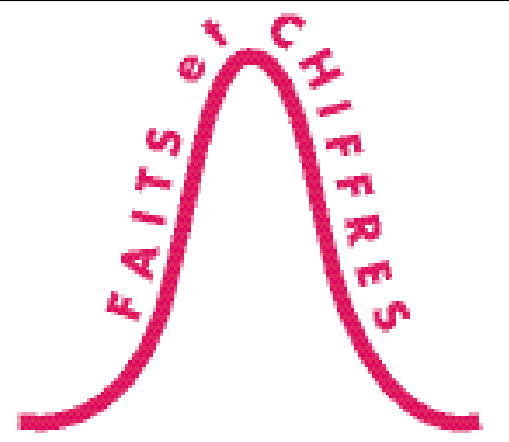

médecine/sciences $1996 ; 12: 1297-1301$

\title{
Le déclin du français médical en Europe et aux États-Unis
}

torsade de pointes, tourniquet, trocart, voyeurisme.

L'hégémonie du français médical culmine dans la seconde moitié du XIX $^{\mathrm{e}}$ siècle. À partir de ce moment, son influence n'a cessé de diminuer: lentement tout d'abord, confrontée au prestige croissant de l'allemand puis de l'anglais, conséquence des deux guerres mondiales qui détruisirent l'Europe et donnèrent naissance à l'ère de la prédominance de la science venue des États-Unis, d'une façon accélérée. C'est ainsi que de moyen universel de communication le français est arrivé en moins d'un siècle à devoir défendre ses positions face à la poussée de l'anglais. Parallèlement, l'apport de la langue de Rabelais au langage médical international a pratiquement cessé et c'est dans la langue anglaise que sont aujourd'hui puisés ces termes en grand nombre: ACTH, by-pass, checkup, clearance, clapping, doping, dumping, feed-back, flutter, immunoblot, lifting, monitoring, odds ratio, pace-maker, rash, scanner, screening, shunt, stripping, test, turnover.

Quoique la tendance vers une importance croissante de l'anglais comme langue internationale de la médecine commence à se dessiner dès la fin de la Première Guerre mondiale, ce n'est qu'après la fin de la Seconde Guerre mondiale qu'elle apparaît clairement. Il est donc fréquent de voir citer les dates de 1945 ou 1950 comme le début de cette étape de prédominance de la langue anglaise.

Malgré la relative proximité dans le temps, nous disposons de peu d'élé- ments objectifs sur le processus par lequel l'anglais s'est converti progressivement en principale langue de la médecine, et encore moins sur l'évolution de l'importance du français dans la médecine occidentale au cours de ce siècle.

\section{Méthodes et résultats}

J'ai récemment publié une étude sur l'évolution des différentes langues dans un journal médical français [4]. Je présente ci-après les résultats d'une étude similaire, mais qui recouvre les principaux pays d'Europe occidentale et les EtatsUnis. Comme on peut le constater au vu du Tableau I, on a déterminé la langue de publication de plus de 135 000 références bibliographiques incluses dans 9050 articles publiés dans huit importantes revues médicales d'Europe et des États-Unis : $L a$ Presse Médicale (France), Schweizerische Medizinische Wochenschrift (Suisse), Deutsche Medizinische Wochenschrift (Allemagne), Wiener Klinische Wochenschrift (Autriche), British Medical Journal (Angleterre), The Journal of the American Medical Association (États-Unis), Nederlands Tijdschrift voor Geneeskunde (Pays-Bas) et Medicina Clínica (Espagne).

Ce tableau présente un résumé des principales caractéristiques méthodologiques des études effectuées dans les huit pays sur lesquels porte l'enquête. La méthode employée et les résultats précis pour les principales langues sont décrites en détail dans les études nationales respectives déjà publiées [5-8].

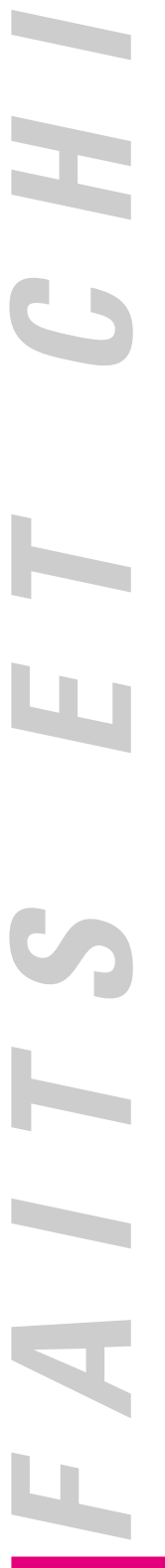


Tableau I

CARACTÉRISTIQUES GÉNÉRALES DE L'ÉTUDE

\begin{tabular}{|c|c|c|c|c|c|c|c|c|}
\hline \multirow{3}{*}{\begin{tabular}{l}
\multicolumn{1}{c}{ Pays } \\
$\begin{array}{l}\text { France } \\
\text { Suisse romande }\end{array}$
\end{tabular}} & \multirow{3}{*}{\begin{tabular}{l}
\multicolumn{1}{c}{ Journal } \\
La Presse Médicale \\
Schweizerische \\
Medizinische Wochenschrifta
\end{tabular}} & \multirow{3}{*}{$\begin{array}{c}\begin{array}{c}\text { Période } \\
\text { étudiée }\end{array} \\
1920-1995 \\
1920-1995\end{array}$} & \multicolumn{2}{|c|}{ Articles $^{c}$} & \multicolumn{2}{|c|}{ Références $^{d}$} & \multirow{3}{*}{$\begin{array}{c}\begin{array}{c}\text { Art./ } \\
\text { année }^{\mathrm{e}}\end{array} \\
50-82 \\
21-76\end{array}$} & \multirow{3}{*}{$\begin{array}{c}\begin{array}{c}\text { Réf./ } \\
\text { année }^{f}\end{array} \\
389-1247 \\
402-1386\end{array}$} \\
\hline & & & 974 & $(80$ & 115 & (11531) & & \\
\hline & & & 756 & (637) & & & & \\
\hline Suisse alémanique & $\begin{array}{l}\text { Schweizerische } \\
\text { MedizinischeWochenschrift }\end{array}$ & 1920-1995 & 1730 & (1434) & 32950 & (32607) & $42-175$ & $1067-2654$ \\
\hline Allemagne & $\begin{array}{l}\text { Deutsche Medizinische } \\
\text { Wochenschrift }\end{array}$ & 1920-1995 & 978 & $(800)$ & 16079 & (16001) & $50-80$ & $344-1417$ \\
\hline Autriche & $\begin{array}{l}\text { Wiener Klinische } \\
\text { Wochenschrift }\end{array}$ & 1920-1995 & 1163 & $(800)$ & 14858 & (14660) & 50-109 & 505-1327 \\
\hline $\begin{array}{l}\text { Angleterre } \\
\text { États-Unis }\end{array}$ & $\begin{array}{l}\text { British Medical Journal } \\
\text { JAMA }\end{array}$ & $\begin{array}{l}1920-1995 \\
1920-1995\end{array}$ & $\begin{array}{r}1088 \\
918\end{array}$ & $\begin{array}{l}(800) \\
(800)\end{array}$ & $\begin{array}{l}10276 \\
12371\end{array}$ & $\begin{array}{l}(10223) \\
(12366)\end{array}$ & $\begin{array}{l}50-119 \\
50-79\end{array}$ & $\begin{array}{l}282-983 \\
227-1717\end{array}$ \\
\hline $\begin{array}{l}\text { Etats-Unis } \\
\text { Pays-Bas }\end{array}$ & $\begin{array}{l}\text { JAMA } \\
\text { Nederlands Tijdschrift voor } \\
\text { Geneeskunde }\end{array}$ & $\begin{array}{l}1920-1995 \\
1930-1995\end{array}$ & $\begin{array}{l}918 \\
776\end{array}$ & $\begin{array}{l}(800) \\
(700)\end{array}$ & $\begin{array}{l}12371 \\
10570\end{array}$ & $\begin{array}{l}(12366) \\
(10462)\end{array}$ & $50-70$ & $342-1024$ \\
\hline $\begin{array}{l}\text { Espagne } \\
\text { Total }\end{array}$ & Medicina Clínica & 1945-1995 & $\begin{array}{r}667 \\
9050\end{array}$ & $\begin{array}{r}(542) \\
(7313)\end{array}$ & $\begin{array}{r}12905 \\
136190\end{array}$ & $\begin{array}{r}(12807) \\
(135143)\end{array}$ & $50-88$ & $805-1507$ \\
\hline
\end{tabular}

a Articles rédigés en français.

${ }^{b}$ Articles rédigés en allemand.

c Nombre total d'articles analysés : entre parenthèses, nombre d'articles contenant au moins une référence bibliographique.

¿ Nombre total de références bibliographiques; entre parenthèses, nombre de références bibliographiques valables (pour lesquelles la langue de publication a pu être déterminée).

e Nombre d'articles analysés pour chaque année étudiée.

${ }^{f}$ Nombre de références bibliographiques valables pour chaque année étudiée.

En ce qui concerne le sujet qui nous occupe, à savoir l'évolution du français, le Tableau II offre un relevé de la fréquence des références en français (exprimée en pourcentage par rapport au total de références), pour chaque pays, pendant la période de temps couverte par l'étude. La figure 1 reprend, pour sa part, l'évolution dans le temps du pourcentage d'articles contenant au moins une référence en français dans les huit pays étudiés.

Il peut être intéressant d'examiner, dans le Tableau II, les valeurs des $5 \%$ et $1 \%$ par rapport au total des références. Ces deux valeurs, quoique pleinement arbitraires, peuvent nous servir d'indicateur, pour la première du moment auquel le français a cessé d'occuper une place prédominante dans le domaine international; pour la seconde, du moment auquel sa présence est devenue purement anecdotique. La dernière année au cours de laquelle le pourcentage de références en français dépassait encore les $5 \%$ a été 1940 en Angleterre et aux États-Unis ; 1960 en Allemagne et aux Pays-Bas ; 1970 en Autriche et en Suisse aléma-

\begin{tabular}{|c|c|c|c|c|c|c|c|c|c|}
\hline \multicolumn{10}{|c|}{$\begin{array}{c}\text { Tableau II } \\
\text { ÉVOLUTION DU POURCENTAGE DE RÉFÉRENCES EN FRANÇAIS } \\
\text { AU COURS DE LA PÉRIODE 1920-1995 DANS LES ARTICLES PUBLIÉS } \\
\text { EN FRANCE (F), EN SUISSE ROMANDE (CHr), EN SUISSE ALÉMANIQUE } \\
\text { (CHa), EN ALLEMAGNE (D), EN AUTRICHE (A), EN ANGLETERRE (GB), } \\
\text { AUX ÉTATS-UNIS (USA), AUX PAYS-BAS (NL) ET EN ESPAGNE (E) }\end{array}$} \\
\hline Année & $\mathbf{F}$ & $\mathrm{CHr}$ & $\mathrm{CHa}$ & D & A & GB & USA & NL & E \\
\hline $\begin{array}{l}1920 \\
1925 \\
1930 \\
1935 \\
1940\end{array}$ & $\begin{array}{l}77,9 \\
66,2 \\
77,0 \\
66,9 \\
56,8\end{array}$ & $\begin{array}{l}61,0 \\
46,0 \\
39,2 \\
48,3 \\
30,9\end{array}$ & $\begin{array}{r}9,1 \\
14,0 \\
9,4 \\
10,6 \\
9,9\end{array}$ & $\begin{array}{l}3,2 \\
4,0 \\
2,4 \\
1,8 \\
2,4\end{array}$ & $\begin{array}{l}7,1 \\
2,2 \\
5,7 \\
5,8 \\
5,0\end{array}$ & $\begin{array}{r}8,9 \\
3,9 \\
11,5 \\
6,9 \\
5,7\end{array}$ & $\begin{array}{l}3,5 \\
8,5 \\
5,5 \\
2,3 \\
6,0\end{array}$ & $\begin{array}{c}- \\
- \\
11,0 \\
13,1 \\
15,6\end{array}$ & $\begin{array}{l}- \\
- \\
- \\
-\end{array}$ \\
\hline $\begin{array}{l}1945 \\
1950 \\
1955 \\
1960 \\
1965 \\
1970\end{array}$ & $\begin{array}{l}77,4 \\
49,4 \\
41,7 \\
50,4 \\
43,9 \\
37,2\end{array}$ & $\begin{array}{l}30,6 \\
28,1 \\
27,0 \\
18,3 \\
23,5 \\
19,8\end{array}$ & $\begin{array}{l}7,2 \\
7,7 \\
7,1 \\
8,6 \\
5,5 \\
5,6\end{array}$ & $\begin{array}{l}2,3 \\
2,3 \\
5,9 \\
5,3 \\
3,7 \\
3,2\end{array}$ & $\begin{array}{l}3,5 \\
3,4 \\
4,9 \\
3,1 \\
4,1 \\
5,2\end{array}$ & $\begin{array}{l}2,0 \\
2,4 \\
3,3 \\
1,8 \\
2,4 \\
1,7\end{array}$ & $\begin{array}{l}1,3 \\
1,3 \\
2,4 \\
0,8 \\
0,6 \\
0,5\end{array}$ & $\begin{array}{l}7,3 \\
7,9 \\
6,5 \\
8,9 \\
3,9 \\
4,8\end{array}$ & $\begin{array}{l}23,1 \\
17,4 \\
22,5 \\
17,8 \\
15,8 \\
17,1\end{array}$ \\
\hline $\begin{array}{l}1975 \\
1980 \\
1985 \\
1990 \\
1995\end{array}$ & $\begin{array}{l}29,6 \\
26,4 \\
21,4 \\
20,5 \\
16,3\end{array}$ & $\begin{array}{r}19,5 \\
12,5 \\
10,8 \\
13,6 \\
6,5\end{array}$ & $\begin{array}{l}4,0 \\
4,3 \\
3,1 \\
2,7 \\
1,5\end{array}$ & $\begin{array}{l}3,0 \\
2,4 \\
0,7 \\
0,2 \\
0,5\end{array}$ & $\begin{array}{l}2,4 \\
0,7 \\
0,3 \\
0,7 \\
0,6\end{array}$ & $\begin{array}{l}0,8 \\
0,6 \\
0,3 \\
0,2 \\
0,1\end{array}$ & $\begin{array}{l}0,9 \\
0,6 \\
0,1 \\
0,0 \\
0,5\end{array}$ & $\begin{array}{l}3,0 \\
3,0 \\
1,6 \\
0,8 \\
0,2\end{array}$ & $\begin{array}{r}12,2 \\
5,9 \\
5,8 \\
2,3 \\
0,5\end{array}$ \\
\hline
\end{tabular}




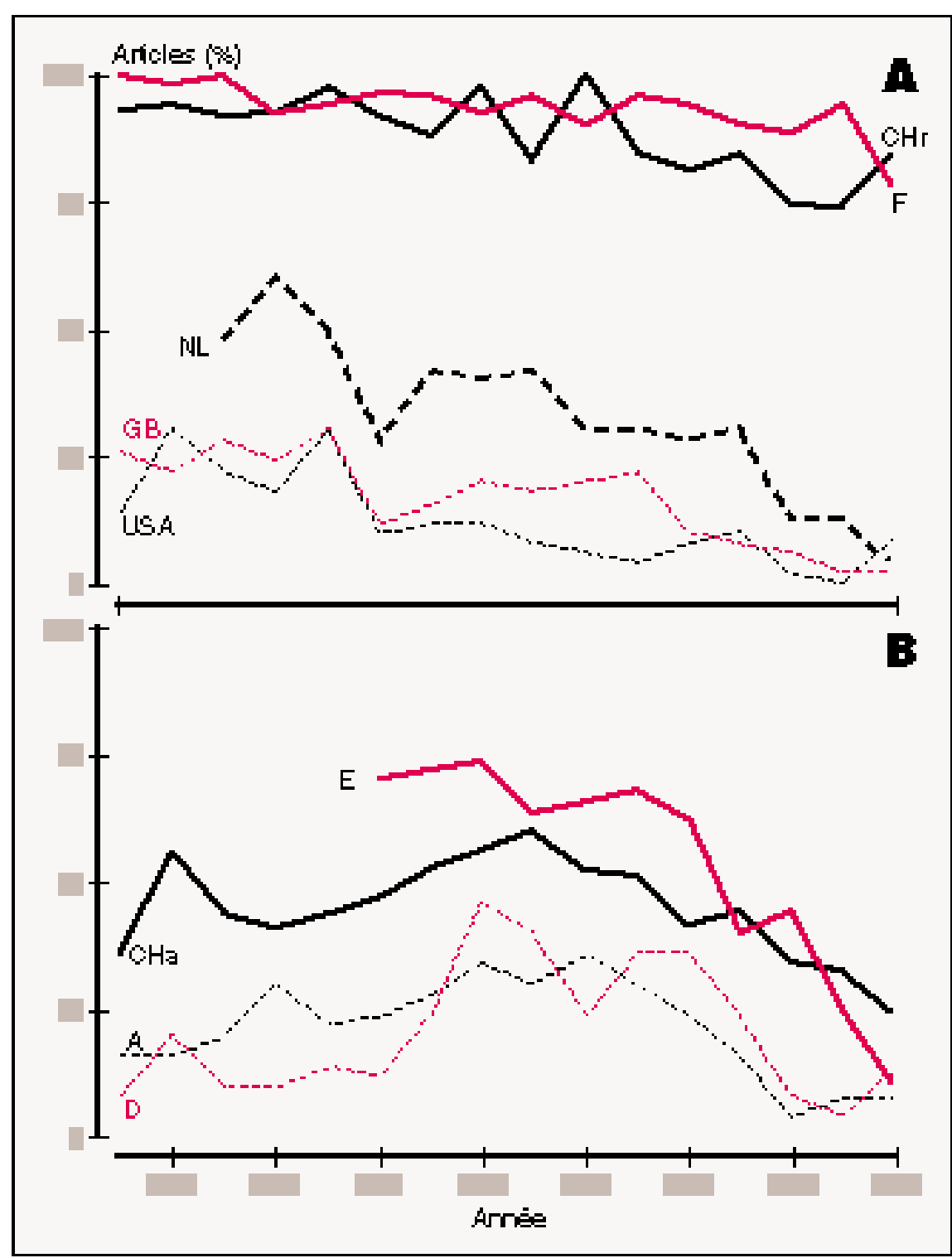

Figure 1. Évolution du pourcentage d'articles contenant au minimum une référence en français pendant la période 1920 à 1995. A : données correspondant à deux pays francophones (F, France; $\mathbf{C H r}$, Suisse romande), aux Pays-Bas (NL) et à deux pays anglophones (GB, Angleterre ; USA, ÉtatsUnis). B : données correspondant à l'Espagne (E) et aux trois pays germanophones (D, Allemagne ; $\mathbf{A}$, Autriche; $\mathbf{C H a}$, Suisse alémanique).

mière année au cours de laquelle le pourcentage de références en français a été inférieur $1 \%$ a été 1960 aux États-Unis ; 1975 en Angleterre ; 1980 en Autriche ; 1985 en Allemagne ; 1990 aux Pays-Bas, et 1995 en Espagne; pour ce qui est de la Suisse alémanique, en 1995 encore $1,45 \%$ des références analysées correspondaient à des publications en français. constante pendant toute la période étudiée. Il est à relever que les pourcentages obtenus en Suisse romande sont étonnamment bas - autour de la moitié de ceux obtenus en France - pour un pays de langue française, ce que l'on pourrait attribuer à la situation minoritaire du français dans un pays qui, comme la Suisse, est à majorité germanophone. Les résultats auraient sans doute été très différents dans d'autres pays francophones bilingues, présentant probablement des pourcentages plus élevés en Belgique francophone et plus faibles au Québec.

2. Dans les pays de langue anglaise (États-Unis et Angleterre), la Seconde Guerre mondiale marque un tournant considérable dans l'importance du français qui, pendant l'entre-deux-guerres (19201940), était cité avec une relative fréquence tant en Angleterre (4$11,5 \%)$ qu'aux États-Unis (2,3$8,5 \%)$. Entre 1940 et 1945 , le pourcentage de références en français diminue dans une proportion de un à quatre aux États-Unis et de près de un à trois en Angleterre. Aux ÉtatsUnis, en passe de devenir dès ce moment la première puissance médicale mondiale, l'importance du français décroît rapidement, et à partir de 1955 sa présence ne sera plus que purement anecdotique $(<1 \%)$. En Angleterre, en revanche, la fréquence des références en français se stabilise autour des $2 \%$ entre 1945 et 1970 ; en 1975 , cependant, elle diminue une fois encore presque de moitié par rapport au niveau qu'elle occupait cinq ans auparavant, sa présence devenant ainsi purement anecdotique.

3. Dans les pays de langue allemande (Allemagne, Autriche et Suisse alémanique) on retrouve les trois phases décrites en Angleterre, quoique moins clairement marquées. Pendant l'entre-deux-guerres (1920-1940), la fréquence des références en français, bien que considérable, est moindre en Allemagne et en Autriche que dans les pays de langue anglaise ; cela paraît logique si nous tenons compte du fait que l'Allemagne était la première puissance médicale de l'époque. Entre 1940 et 1945 la chute du français est moins marquée que dans les pays de langue anglaise, et de 1945 à 1970 le

(n) 
français non seulement se stabilise, mais arrive même à dépasser les pourcentages de 1940 dans les trois pays germanophones. Ce comportement, imputable sans doute au fait que l'allemand perd sa position hégémonique, est plus frappant en Allemagne, où les pourcentages de références en français en 1955 et 1960 dépassent de plus du double ceux obtenus en 1930, 1935 ou 1940. A partir de 1970 , la chute du français est très évidente en Allemagne et en Autriche ; à titre d'exemple, le pourcentage de références en français passe en Autriche de $5,2 \%$ en 1970 à $2,4 \%$ en 1975 , $0,8 \%$ en 1980 , et $0,3 \%$ en 1985 (près de 15 fois moins qu'en 1970 !). Au cours de toute la période étudiée, le pourcentage de références en français a été supérieur en Suisse alémanique que dans le reste des pays de langue allemande, ce qui semble logique si nous tenons compte du fait que le français est la deuxième langue nationale suisse.

4. Aux Pays-Bas on distingue aussi clairement les trois mêmes étapes déjà mentionnées, avec des pourcentages supérieurs à $10 \%$ jusqu'en 1940, une forte chute entre 1940 et 1945, un maintien de l'importance du français entre 1945 et 1970, une baisse importante à partir de cette date, et une quasi-disparition au cours des dernières années.

5. Pour l'Espagne, où nous ne disposons que des chiffres correspondant aux deux dernières étapes citées (la revue Medicina Clinica n'ayant été fondée qu'en 1943), les pourcentages obtenus sont étonnamment élevés, oscillant entre $15 \%$ et $25 \%$ jusqu'en 1970. A partir de cette date, le pourcentage des références diminue rapidement, pour se cantonner dans des valeurs insignifiantes en 1995. Les résultats obtenus en Espagne mettent en évidence l'extraordinaire influence que le français a exercé sur les pays latins jusqu'à des dates relativement récentes, fait fréquemment ignoré par les Français eux-mêmes.

\section{Le français médical : décadence en trois étapes}

En résumé, on peut distinguer dans les grandes lignes trois étapes dans l'évolution de l'importance du fran- çais en médecine au cours du $\mathrm{XX}^{\mathrm{e}}$ siècle : la première allant jusqu'à 1940 ; la seconde qui couvre la période comprise entre 1945 et 1970 ; la troisième qui a débuté en 1975 et ne semble pas avoir encore pris fin.

- Première étape. Jusqu'en 1940, le français n'est pas seulement la langue indiscutée de la médecine en France, mais également, conjointement avec l'allemand et l'anglais, l'une des trois grandes langues internationales de la médecine.

- Deuxième étape. Entre 1940 et 1945 on assiste à une chute sévère dans l'importance mondiale du français, conséquence de la Seconde Guerre mondiale qui dévaste l'Europe, anéantit les laboratoires français, anglais et allemands et déplace le centre de la médecine vers les États-Unis. Entre 1945 et 1970, l'importance de l'anglais continue à croître dans tous les pays étudiés, mais essentiellement aux dépens de l'allemand et de la langue nationale de chaque pays, raison pour laquelle pendant cette période le français réussit à maintenir (voire à accroître légèrement) son importance hors des pays de langue française. En 1970, par exemple, le pourcentage d'articles contenant au moins une référence en français était encore de $22 \%$ en Angleterre, de $30 \%$ à $38 \%$ aux Pays-Bas, en Autriche et en Allemagne, de $52 \%$ en Suisse alémanique et de $70 \%$ en Espagne.

- Troisième étape. A partir de 1975 , on perçoit une chute accélérée de l'importance du français, tant en France qu'à l'extérieur du pays. En 1995, par exemple, le pourcentage des références en français dépasse à peine les $16 \%$ en France, et se voit relégué à des valeurs pratiquement anecdotiques à l'étranger ; même en Espagne, où jusqu'en 1975 des références en français figuraient dans plus de la moitié des articles, le pourcentage de celles-ci a baissé en 1995 jusqu'au niveau du catalan! A quoi obéit ce déclin prononcé du français en tant que langue médicale internationale de la médecine au cours des deux dernières décennies ? On l'attribue le plus souvent à la prédominance des États-Unis en matière médicale, mais cela ne semble guère probable, car cette prédominance a débuté dès 1945 , alors que, comme nous venons de le voir, le français a réussi à se maintenir sur ses positions, sur le terrain international, jusqu'en 1970. Il est fort probable que la véritable cause de cette récente décadence du français soit de type interne. A partir de 1975, alors que la suprématie mondiale de la science américaine est déjà internationalement acceptée et que l'anglais se voit par conséquent élevé au rang de langue internationale de la médecine, le nombre d'auteurs de langue française publiant leurs travaux en anglais, que ce soit dans des revues internationales ou même françaises, va croissant. Ce phénomène semble devoir prendre toujours plus d'ampleur et affecter principalement les auteurs les plus éminents et les travaux scientifiques ou cliniques d'une importance majeure, non seulement dans les pays de langue française, mais également dans le reste des pays non-anglophones. Quoiqu'il s'agisse d'un phénomène quasiment inexploré, Maher [9] présente quelques chiffres significatifs : en 1970 encore, seuls 3,1\% des articles publiés en France et répertoriés dans l'Index Medicus étaient rédigés en anglais ; en 1980 ce pourcentage atteignait déjà les $11,7 \%$. Une autre donnée citée par Devanlay [10] va dans le même sens : en 1985, 56,1 \% des 14635 articles publiés par des auteurs français et répertoriés dans la section science de la vie du SCI étaient rédigés en anglais.

A en juger par le nombre élevé de commentaires parus dans les principaux journaux médicaux de France, du Québec, de Belgique et de Suisse romande, les médecins francophones assistent avec préoccupation à ce déclin généralisé du français. En termes généraux, l'on peut distinguer deux points de vue opposés qu'il convient tout au moins - quoique le moment ne se prête pas à une analyse détaillée - de mentionner en passant:

En premier lieu, un groupe de médecins juge nécessaire de défendre la langue française et de continuer à publier des textes en français, quoique - il est vrai - seulement des articles de grande qualité [11-13]. C'est ainsi que l'on verra fréquemment citer médecine/sciences 
comme exemple d'une revue francophone de qualité. Les partisans de ce point de vue considèrent que l'usage du français ne doit pas être un obstacle à la diffusion internationale d'une publication. En faveur de cette thèse, nous pouvons citer les données obtenues par Locquin [14], qui distribua en 1983 un questionnaire à 3000 scientifiques de 50 pays, afin de connaître le degré de compréhension des différentes langues au sein de la communauté scientifique internationale. D'après ses résultats, $77 \%$ des scientifiques consultés étaient capables de comprendre le français, d'où Locquin déduit que le français est prodigieusement sous-utilisé par rapport aux possibilités mondiales de sa compréhension.

D'autres sont d'avis, au contraire, que c'est moins la langue française que la science française (ou québécoise) qui est en danger, et que c'est cette dernière qu'il faut défendre, même en publiant en anglais [15-17]. Ces auteurs considèrent que la langue française ne va ni disparaître, ni perdre de son importance pour avoir vu les scientifiques et médecins publier leurs articles en anglais. L'affirmation suivante de Moziconacci résume bien cette position : «Une découverte originale ne peut prendre rang dans la communauté scientifique que si elle a paru dans une grande revue anglo-américaine " [15]. Comme il est logique de le supposer, les auteurs américains partagent cette opinion [18].

Comme c'est le cas pour toute controverse, divers arguments peuvent être allégués en défense de l'une ou l'autre thèse, et dans des occasions très similaires. Pour citer un exemple récent, il est indubitable que si l'équipe de Montagnier arrivait à développer un vaccin efficace contre le SIDA, sa découverte, même publiée en français, serait immédiatement connue aux quatre coins du monde [19]. En même temps, on peut dire que le célèbre affrontement entre Montagnier et Gallo pour s'accorder la primauté de la découverte du virus du SIDA aurait probablement trouvé une issue bien différente si le groupe de l'Institut Pasteur avait publié ses travaux exclusivement en français.
Les résultats que j’ai présentés donnent une large vision du déclin du français dans la médecine occidentale, mais beaucoup d'éléments nous échappent encore. Dans le domaine de la francophonie, par exemple, il conviendrait d'étudier quelle a été l'évolution du français dans au moins un pays d'Afrique francophone, ainsi que dans d'autres pays bilingues, tels la Belgique ou le Canada. En ce qui concerne les pays latins, il est fort possible que la situation en Espagne présente des différences considérables avec celle prévalant au Portugal, en Italie, en Roumanie ou en Amérique latine. Toujours en Europe occidentale, les données nous manquent concernant la situation du français dans les pays scandinaves. Plus rares encore sont celles dont nous disposons concernant les pays qui échappent à la zone d'influence des Etats-Unis, c'est-à-dire la Russie et les pays de l'ancienne zone soviétique d'une part, et le Japon d'autre part. C'est ainsi qu'un vaste terrain inexploré s'offre encore aux chercheurs intéressés par l'étude de l'évolution du français comme langue internationale de la médecine

\section{RÉFÉRENCES}

1. Dirckx JH. French and German words in medical English. Am J Dermatopathol 1989 ; $11: 392-5$.

2. Navarro FA. Palabras francesas de traducción engañosa en medicina. Med Clín (Barc) 1996 ; $106: 417-26$.

3. Murken AH. Rückblick auf die historische entwicklung der medizinischen fachsprache. Dans : Lehrbuch der medizinischen terminologie, $3^{\mathrm{e}}$ ed. Stuttgart: Wissenschaftliche Verlagsgesllschaft, $1994 ; 15-26$.

4. Navarro FA. L'importance de l'anglais et du français sur la base des références bibliographiques de travaux originaux publiés dans La Presse Médicale (1920-1995). Presse Med 1995 ; 24 : 1547-51.

5. Navarro FA. De taal in de geneeskunde afgeleid uit literatuurreferenties van oorspronkelijke stukken in het Nederlands Tijdschrift voor Geneeskunde (1930-1995). Ned Tijdschr Geneeskd 1996 ; 140 : 1263-7.

6. Navarro FA. Die Sprache der Medizin in Österreich (1920-1995). Wien Klin Wochenschr $1996 ; 108: 363-9$.
7. Navarro FA. El idioma de la medicina a través de las referencias bibliográficas de los artículos originales publicados en medicina clínica durante 50 años (1945-1995). Med Clin (Barc) 1996 (sous presse).

8. Navarro FA. Englisch oder Deutsch ? Die sprache der medizin in Deutschland von 1920 bis 1995. Dtsch Med Wochenschr 1996 (sous presse).

9. Maher J. The development of English as an international language of medicine. Applied Ling 1986 ; 7 : 206-18.

10. Devanlay A. Lingua franca. Pédiatrie $1992 ; 47: 5-7$.

11. Hauteville D. A propos de l'usage de l'anglais dans les publications scientifiques françaises. Ann Med Intern $1995 ; 146: 29$.

12. Zucman J. Y a-t-il encore une place pour une littérature orthopédique non anglosaxonne? Rev Chir Orthop Repar Appar Mot $1992 ; 78: 275-8$.

13. Blancher G, Sournia JC. Médecine et langue française. Bull Acad Natl Med 1994 ; $178: 227-32$

14. Locquin MV. La langue française dans les sciences biologiques et médicales. Bull Acad Natl Med 1983 ; 167 : 41-8.

15. Mozziconacci P. Faut-il défendre la langue française? Plaidoyer pour le bilinguisme. Ann Pediatr (Paris) 1990 ; 37 : 419-20.

16. Balibar S. L'anglais pour défendre la science française ? La Recherche 1992; 23 : 1068-9.

17. Bonfils S. Défense du francais ou promotion de la francité ? Gastroenterol Clin Biol 1981 ; 5 : 179-82.

18. Garfield E. Multilingual communication essential for science. Interdiscip Sci Rev 1995 ; $20: 179-80$

19. Decaux A. Le combat du francais scientifique n'est pas perdu d'avance. Quot Med $1990 ; 9$ janvier : 23.

\section{Fernando A. Navarro}

Service de traduction médicale, (PSDU), F. Hoffmann - La Roche SA, CH-4070 Bâle, Suisse et Wilhelm His-Strasse 5, 1. Stock, CH-4056 Bâle, Suisse. e-mail : fernando.navarro@roche.com

\section{TIRÉS À PART}

\section{F.A. Navarro.}

